\title{
Uma Experiência na Criação de Game Design de Jogos Digitais Educativos a partir do Design Thinking
}

\begin{abstract}
Kleber Tavares Fernandes - PPgSC / UFRN - kleber76@gmail.com Márcia Jacyntha Nunes Rodrigues Lucena - DIMAP / UFRN - marciaj@ dimap.ufrn.br Eduardo Henrique da Silva Aranha - DIMAP / UFRN - eduardoaranha@dimap.ufrn.br
\end{abstract}

Resumo: A possibilidade de criação de seus próprios jogos motivam os alunos a desenvolverem o pensamento computacional e aprenderem os conteúdos curriculares envolvidos nesse processo. Entretanto, para obter um bom jogo educativo, é necessário conceber um bom Game Design. Sendo assim, a escolha de estratégias que dão suporte a elaboração do Game Design do jogo mostra-se determinante. Este artigo apresenta um relato sobre a utilização de uma proposta de concepção de Game Design de jogos digitais educativos, baseada no Design Thinking. O objetivo do trabalho é identificar como essa proposta pode estimular momentos pedagógicos significativos para a aprendizagem. Os resultados apontam que a proposta motiva os alunos a aprenderem mais sobre o processo de desenvolvimento e os assuntos abordados pelos jogos.

Palavras-Chave: Game Design, Jogos Educativos, Design Thinking

\section{An Experience in Creating Game Design of Serious Games from Design Thinking}

Abstract. The possibility of creating their own games motivate students to develop computational thinking and to learn the curricular contents involved in this process. However, to get a good serious game, you need to design a good Game Design. Therefore, the choice of strategies that support the elaboration of Game Design of the game is decisive. This article presents an account about the use of a Game Design design proposal for serious games, based on Design Thinking. The objective of this work is to identify how this proposal can stimulate significant pedagogical moments for learning. The results point out that the proposal motivates students to learn more about the development process and the issues addressed by the games.

Keywords: Game Design, Serious Game, Design Thinking

\section{Introdução}

Os recursos tecnológicos aplicados na educação têm possibilitado que os alunos interajam de maneira mais dinâmica, participativa e significativa com os conteúdos ministrados em sala de aula. $\mathrm{O}$ uso de estratégias inovadoras os tem motivado a um maior engajamento, despertando sua curiosidade e ampliando as possibilidades de aprendizagem.

Essa pode ser uma saída para o que Prensky (2001) afirmou em sua pesquisa: "Cada vez menos os alunos se adequam ao sistema educacional que está posto, dada a incompatibilidade entre suas características de aprendizagem e os métodos convencionais aplicados em muitas escolas".

Ao pensarmos em novas estratégias de ensino devemos considerar também o uso das novas tecnologias como uma tentativa de tornar o processo de aprendizagem mais 
significativo e mais próximo da geração desses alunos. Segundo Prensky (2010) existe uma real necessidade da criação de abordagens para educação mais adequada a esta geração, por exemplo, o uso de jogos digitais.

O uso e o desenvolvimento de jogos na educação é uma das possibilidades para explorar o conhecimento, possibilitando aos alunos aprenderem enquanto jogam (Silva, 2017).

Para Savi e Ulbricht (2008) o ato de jogar facilita a aprendizagem por favorecer o desenvolvimento da atenção, da concentração, do raciocínio lógico, da investigação, da socialização, da coordenação motora e do desenvolvimento das habilidades cognitivas. A utilização de jogos digitais na educação possibilita não somente a modernização do processo de ensino-aprendizagem, como também instiga cada vez mais os alunos a buscarem e a participarem da construção do seu conhecimento. Oferecendo a eles a oportunidade de serem elementos ativos no processo de aprendizagem (Nogueira; Galdino, 2012).

Há relatos não apenas do uso, mas também do desenvolvimento de jogos digitais por alunos como estratégia de ensino aprendizagem (Marinho et al. 2011). A possibilidade de criação de seus próprios jogos motivam os alunos a desenvolverem o pensamento computacional e aprenderem os conteúdos curriculares envolvidos nesse processo.

Entretanto, o desenvolvimento de um jogo digital não é uma tarefa trivial. Segundo Chandler (2012), o processo de produção de um jogo inicia com a definição do seu conceito inicial (Game Design) e termina com a criação de uma versão final do jogo, sendo que várias etapas acontecem entre esses dois pontos. Portanto, para obter um bom jogo digital, é necessário conceber um bom Game Design. A escolha de estratégias que dão suporte a elaboração do Game Design do jogo mostra-se determinante.

Outra abordagem que vem sendo utilizada na educação é o Design Thinking, principalmente nos processos de aprendizagem baseada em problemas. Essa abordagem possui etapas bem definidas: delimitação do problema, pesquisa do público-alvo, geração de ideias, prototipagem de possibilidades, seleção de soluções e implementação do produto.

Aliar, portanto, a elaboração de Game Design de jogos digitais utilizando as técnicas do Design Thinking pode ser uma estratégia interessante no processo de ensinoaprendizagem.

Este artigo apresenta um relato sobre a utilização de uma proposta de concepção de Game Design de jogos digitais educativos, baseada nos conceitos do Design Thinking. O objetivo do trabalho é identificar como essa proposta pode estimular momentos pedagógicos significativos para a ensino-aprendizagem. Os dados foram obtidos através de observações e entrevistas com os participantes de oficinas de criação de jogos. Os resultados apontam que a metodologia proposta motiva os alunos a aprenderem mais sobre o processo de desenvolvimento e os assuntos abordados pelos jogos.

Na seção seguinte, destacamos os conceitos de Game Desing e Design Thinking, bem como os respectivos autores que subsidiaram a elaboração da abordagem proposta para realização da experiência relatada. Em seguida, apresentamos o processo de elaboração dessa abordagem. $\mathrm{Na}$ seção 4, fazemos o relato da experiência de uso da 
abordagem proposta através de uma oficina de criação de jogos digitais educativos. $\mathrm{Na}$ seção 5, apresentamos os trabalhos relacionados. Por fim, tecemos nossas considerações finais, reconhecendo a pertinência da proposta e o seu uso na educação.

\section{Fundamentação Teórica}

Esta seção apresenta os principais conceitos utilizados neste trabalho: Game Design e Design Thinking.

\subsection{Game Design}

Antes de produzirmos efetivamente um jogo, em sua fase de desenvolvimento, é necessário pensarmos na sua ideia, seus requisitos e em todos os seus elementos. Nessa etapa do projeto do jogo, chamada de Game Design, são definidos: sua ideia central, estilo, público alvo, narrativa, mecânica, personagens, cenários, objetos, fases, níveis de dificuldade, sonorização, entre outros. Entretanto, sendo um jogo educativo é necessário também definirmos seus aspectos pedagógicos.

Segundo Mastrocola (2015), o Game Design envolve um contexto para ser vivenciado pelo jogador do qual emerge significado. Para Brathwaite e Schreiber (2009), Game Design é o processo de criar o conteúdo e as regras de um jogo; é o processo de criar objetivos que o jogador sinta-se motivado a alcançar e regras que este siga como se estivesse tomando decisões significativas para atingir esses propósitos.

Na visão de Adams e Rollings (2007), Game Design é a essência de um jogo. Define como ele funciona, descreve seus elementos e é elaborado com a participação de diferentes profissionais da equipe que irá produzi-lo para certo tipo de público de jogadores.

Portanto, podemos compreender o Game Design como um processo complexo, que permite a visualização de todo o conjunto do trabalho a ser desenvolvido pela equipe de produção de jogos (Arruda, 2014).

Pensar em levar o processo de produção do Game Design de um jogo educativo para sala de aula é imaginar diferentes possibilidades de aprendizagem, já que envolve diversos conhecimentos que podem estar relacionados aos conteúdos curriculares. Outro aspecto importante é a participação do educador como um articulador e facilitador do desenvolvimento das atividades que estão envolvidas na produção de um jogo educativo. A assimilação das técnicas necessárias para essa produção é possível e necessária em tempos cada vez mais imersos em uma realidade tecnológica que permeia todas as áreas do conhecimento.

\subsection{Design Thinking}

Originalmente concebida por Nigel Cross, o Design Thinking é um método para resolver problemas baseado em soluções. Em vez de se iniciar com um problema, iniciase com uma solução-base e em seguida definem-se parâmetros para se atingir o objetivo final (Cross, 2011). 
Design Thinking pode ser definido como uma abordagem para solucionar problemas através do conhecimento do seu contexto e propostas de soluções. Coloca as pessoas no centro do desenvolvimento de um projeto, explorando sua criatividade e participação para geração de soluções. Prioriza o trabalho colaborativo em equipes multidisciplinares em busca de soluções inovadoras (Lockwood, 2009).

Segundo Brown (2010), Design Thinking integra o que é desejável do ponto de vista humano ao que é tecnológica e economicamente viável, sendo possível aplicar técnicas de design a uma ampla gama de problemas.

Partindo desses conceitos iniciais é possível imaginar que a abordagem do Design Thinking pode também ser utilizada como estratégia de ensino-aprendizagem, visto que propõe a resolução de problemas contextualizados, por equipes multidisciplinares e que interagem com diversos conteúdos escolares. Além disso, estimula o pensamento crítico, a tomada de decisão, a colaboração, a liderança, a iniciativa, o empreendedorismo, a comunicação oral e escrita, a curiosidade e a imaginação. Esses temas também estão presentes (ou deveriam estar) nos ambientes de sala de aula.

O Educadigital (2013) mostra que a aplicação da abordagem na educação se dá a partir de momentos de descoberta, interpretação, ideação, experimentação e evolução. A descoberta é o momento que percebemos que temos um desafio para enfrentar. Observar as pessoas envolvidas no desafio e entender suas necessidades. É preciso entender o desafio, saber como abordá-lo e reunir inspirações para vencê-lo. A interpretação é a fase seguinte que consiste em procurar entender os significados do desafio. Em seguida, é necessário gerar ideias e oportunidades para solucionar tal problema. $\mathrm{Na}$ fase de experimentação são gerados protótipos da ideia de solução concretizada. Obtém-se o feedback dos envolvidos para saber se a ideia resolve o desafio. Por fim, a evolução propõe aprimorar a solução idealizada.

A abordagem proposta nesse trabalho é uma adaptação do Design Thinking para Educadores, do Educadigital (2013), como estratégia para criação do Game Design de jogos digitais educativos e que será apresentada na seção seguinte.

\section{Construção da abordagem proposta}

Como já explicado anteriormente, o processo de desenvolvimento de um jogo exige a presença de uma equipe multidisciplinar, assim como também prevê o Design Thinking. De maneira colaborativa a equipe interage e expõe suas ideias através de quadros de tarefas, canvas e/ou mapas conceituais. Protótipos são elaborados com a intensão de validar as ideias, com os seus próprios usuários, ainda durante o processo de produção do jogo.

Partindo desse contexto e com base nas experiências vivenciadas pelos autores em projetos executados num laboratório de pesquisas em games e educação de uma Universidade Federal Brasileira, foi elaborada a abordagem proposta, aqui chamada de Creative Game Design - CGD, que é dividida em quatro fases: imersão, ideação, concepção e validação, conforme ilustrado na Figura 1. 


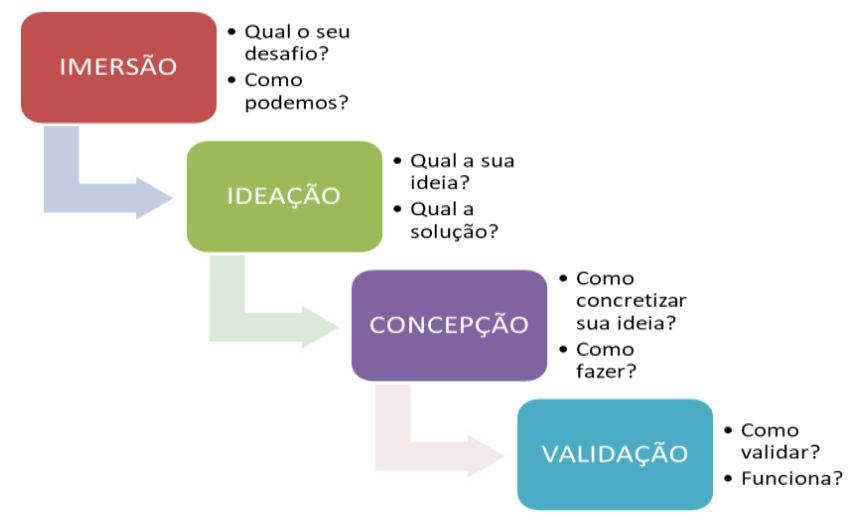

Figura 1 - Fases do Creative Game Desing

Para facilitar as discussões, o registro e a apresentação das informações em cada fase da abordagem são utilizados alguns Templates previstos pelo CGD. A coleção desses Templates constitui o registro documental do projeto do jogo, conhecido em outras abordagens como Document Game Design. Todos os documentos podem ser acessados em https://goo.gl/JBrRp1.

$\mathrm{Na}$ fase de Imersão a equipe define que desafio/problema será abordado pelo jogo. Nessa fase, a equipe busca conhecer o contexto e delimita o problema que será tratado pelo jogo. Estuda o público alvo e suas necessidades e pode consultar especialistas para entender melhor o desafio. Para tanto, utiliza as técnicas de entrevista, observação e pesquisa de campo para levantar esses dados.

A fase de Ideação trata das ideias de solução para o desafio. Qual a ideia do jogo? A equipe pensa em possibilidades de solução, seleciona e descreve uma ideia promissora. São utilizadas técnicas de Brainstorming para escolha da melhor ideia do grupo para o jogo.

Já na Concepção a equipe discute como concretizar a ideia do jogo escolhida na fase anterior. Nessa fase, são definidas algumas características do jogo, tais como: espaço, tempo, regras, mecânica, personagens, cenário, fluxo, fases, aspectos de comunicação e interação, entretenimento, acessibilidade, usabilidade e os aspectos pedagógicos (componente curricular, objetivo, conteúdos, habilidades trabalhadas, entre outros). Também deve ser criada a narrativa do jogo. Essa fase contempla um conjunto de 6(seis) Templates que auxiliam na discussão e registro das informações.

A última fase é a Validação onde são avaliados e validados os projetos dos jogos. Essa fase envolve, além da equipe, professores, especialistas e o usuário final. A equipe apresenta o projeto do jogo através de um Pitch para a equipe de avaliadores. Estes fazem as observações de melhoria, caso necessário, e registam o feedback no Template específico da fase.

Completadas todas as fases do CGD, tem-se o Game Design do jogo concluído e registrado nos Templates elaborados. Parte-se então para o desenvolvimento do jogo e demais etapas do seu processo de produção.

Com o objetivo de tornar prática a experiência de uso do CGD, a seguir é apresentada a oficina de criação de jogos educativos desenvolvida durante um evento voltado para a temática de jogos e educação, realizada por uma Universidade Federal Brasileira. 


\section{Oficina de criação de jogos digitais educativos}

Esta oficina foi uma das oportunidades de experimentação da abordagem CGD durante a realização de um evento relacionado a jogos e educação. Cerca de 50 participantes ( $72 \%$ do sexo masculino e $28 \%$ do sexo feminino), alunos de graduação $(86 \%)$, pós-graduação $(4 \%)$ e professores $(10 \%)$ de diversos cursos de tecnologia da informação e educação (20\% do total dos participantes com experiência em desenvolvimento de jogos), foram desafiados a criarem o Game Design de um jogo educativo. Inicialmente foram apresentados os objetivos da oficina, alguns conceitos iniciais sobre o processo de desenvolvimento de jogos e Game Design, como também a formação das equipes de trabalho (baseada na experiência dos participantes). As fases do CGD foram apresentadas de maneira prática à medida que a oficina era realizada. As Figuras 2 e 3 ilustram alguns momentos da oficina.

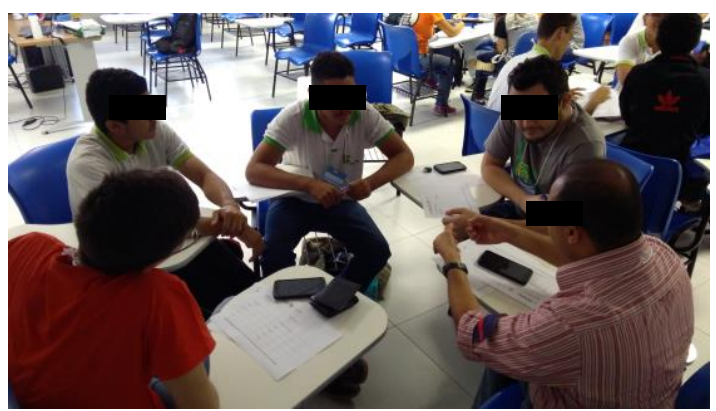

Figura 2 - Momentos de produção

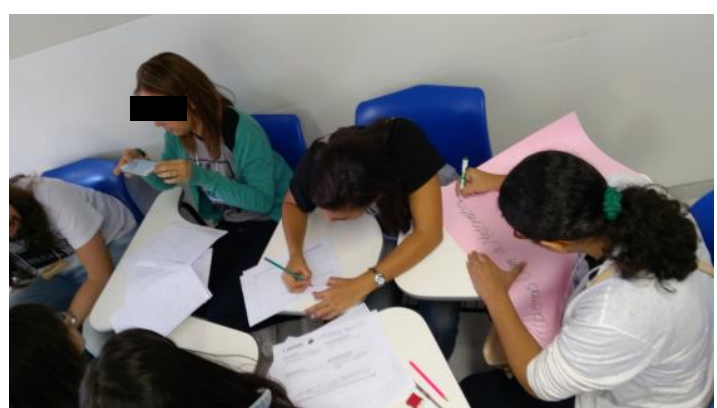

Figura 3 - Momentos de produção

Em seguida, as equipes pensaram em problemas relacionados a um ou mais componentes curriculares (problema educacional) como desafio, cuja solução se daria na forma de um jogo educativo. Por exemplo, o problema de compreensão da ordem numérica na matemática. Ou seja, desafios como esse foram a motivação para concepção de um jogo. Essa atividade foi realizada na fase de Imersão, onde as equipes buscaram conhecer o contexto e o público alvo que envolve o problema.

Já na fase de Ideação, as equipes pensaram em possibilidades de solução para o desafio e escolheram a ideia mais promissora. Para o exemplo anterior a equipe decidiu fazer um jogo de corrida onde o usuário teria que ordenar corretamente os elementos. Desenvolveram a ideia na fase de Concepção, definindo as principais características e elementos do jogo, assim como explicado na seção anterior. Por último, cada equipe produziu um Pitch apresentando o projeto elaborado. As demais equipes validaram as ideias de cada jogo apresentado na fase de Validação.

Em cada fase, eram respondidas as questões presentes nos Templates do CGD. Ao final, o conjunto desses Templates formou o Game Design do jogo proposto.

Por fim, os participantes da oficina responderam um questionário avaliativo de múltipla escolha, elaborado pelos autores através de pesquisas que abordavam a concepção de Game Design de jogos. A análise dos dados coletados apresentou os seguintes resultados:

- $100 \%$ dos participantes afirmaram que as informações apresentadas durante a oficina foram suficientes para a produção do Game Design do jogo.

- $96,7 \%$ consideraram que os Templates facilitaram a elaboração da documentação do jogo. Desses, $50 \%$ afirmaram não ter tido dificuldade em responder as 
questões apresentadas, 36,7\% tiveram dificuldades parciais e 13,3\% sentiram dificuldade de interpretação das perguntas.

- Nenhum participante sentiu falta de algum item do Game Design que não foi contemplado pelos Templates.

- $86,7 \%$ afirmaram que o trabalho colaborativo facilitou o desenvolvimento do Game Design do jogo. 13,3\% tiveram dificuldade de gerenciar o trabalho em grupo.

- $\quad 96,7 \%$ consideraram que o CGD facilitou a produção do Game Design do jogo.

- $\mathrm{Na}$ fase de validação, as equipes avaliaram os projetos dos jogos. 43,3\% receberam o conceito muito bom, $36,7 \%$ bom e $20 \%$ regular. Percebemos que $80 \%$ dos projetos foram bem avaliados (bom e muito bom).

- $100 \%$ consideraram que a abordagem CGD pode ser utilizada por professores e alunos em sala de aula de qualquer segmento de ensino.

Analisando os dados da própria tabulação do questionário avaliativo, é possível inferir a validade da abordagem proposta, uma vez que $100 \%$ dos participantes afirmaram terem obtido informações suficientes para produzir o Game Design do jogo e sequer sugeriram novas questões para os Templates. Além disso, questionamos os participantes que possuem experiência em desenvolvimento de jogos digitais sobre a completude do CGD. Estes afirmaram ser suficiente para a elaboração dos jogos, inclusive sendo de fácil compreensão.

Além do questionário, os pesquisadores observaram o trabalho realizado durante toda oficina. Percebemos um grande envolvimento das equipes nas atividades e a preocupação em especificar os requisitos necessários para produção dos jogos.

No decorrer das fases do CGC, notamos que as equipes propuseram soluções criativas para os desafios elencados. Observamos também uma grande preocupação em especificar corretamente os requisitos pedagógicos do jogo, visando atender as necessidades de aprendizagem do público alvo.

Verificamos a necessidade de ampliação do tempo necessário para realização das atividades de cada fase do CGC, de modo que cada uma delas possa ser executada por completo, de maneira ágil e que traga melhores resultados.

Outra estratégia de avaliação da oficina foi a reunião do grupo de professores presentes ao final da atividade para que os mesmos relatassem a experiência vivenciada e as possibilidades de uso do CGD em sala de aula. Eles relataram que é possível envolver, sem muitas dificuldades, os conteúdos dos diversos componentes curriculares no processo de produção do Game Design dos jogos, sendo possível aplicar tanto na educação básica, como no ensino superior.

\section{Trabalhos relacionados}

Com base em estudos e pesquisas, selecionamos alguns trabalhos que estão relacionados a este.

Murakami et al. (2014) apresentaram o emprego da metodologia Design Thinking devidamente adaptada à criação de jogos educativos, apontando vantagens e desvantagens na comparação com métodos usuais. Depois de estabelecidas as regras gerais do jogo as atividades eram distribuídas pela equipe que não possuíam um local 
fixo para o trabalho. A comunicação era feita através de e-mail e o produto final gerado pela metodologia é um documento de Game Design (GDD).

Silva e Bittencourt (2017) apresentaram uma proposta de metodologia para o ensino e o desenvolvimento de jogos digitais baseada em Design Thinking. A metodologia contempla quatro etapas principais: formação da equipe, concepção do jogo, prototipação e validação. Eles afirmaram que o Design Thinking podem agregar, de forma significativa, no sentido de criatividade, inovação e inspiração às diferentes etapas de projeto de um jogo.

Jewell (2016) usa o termo game Design Thinking como sinônimo de gamificação e discute o uso deste no desenvolvimento de jogos sérios e aplicações no ensino superior e em empresas.

Vargas (2015), em sua dissertação de mestrado, apresenta uma extensão do Design Thinking Canvas com foco em modelos de negócio para a indústria de games. $\mathrm{O}$ objetivo do trabalho é elaborar um novo modelo de documento (Canvas) que auxilia na concepção de jogos.

Martins, Xavier e Dias (2017) apresentaram o Game Design Thinking como proposta lúdica de letramento digital infantil. As etapas da proposta são: empatia, análise e síntese; ideação; prototipação e teste. Segundo os autores, o estímulo à criatividade, escrita e a auto expressão foi a grande contribuição do trabalho.

Silva et al. (2016) descrevem uma experiência de uso do Design Thinking aplicada no contexto de um problema educacional a fim de buscar soluções mais adequadas. Utilizou as etapas comuns do Design Thinking. Verificou-se que o processo sistemático e criativo de design permitiu uma evolução gradativa da compreensão do problema em estudo rumo à solução mais eficiente.

Apesar das experiências relatadas nos trabalhos listados, percebe-se ainda uma carência de metodologias que auxiliem comprovadamente no processo de ensino aprendizagem, tanto da área de criação de jogos digitais como também de conteúdos curriculares do ensino regular. A quantidade e qualidade dessas experiências ainda não são suficientes para afirmamos sobre sua efetividade na educação. Portanto, faz-se necessário investirmos mais sobre esse assunto.

Nossa proposta difere das demais por apresentar etapas enxutas e objetivas. Principalmente por associar a essas etapas o preenchimento dos Templates já apresentados nas seções anteriores, o que facilita o trabalho das equipes.

\section{Considerações Finais}

Sabemos que muitos trabalhos evidenciam a importância do uso e desenvolvimento de jogos como estratégia de ensino-aprendizagem por oferecer ao aluno a oportunidade de ser um agente ativo da construção do seu conhecimento (Sutherland, 2014) e (Savi et al. 2014).

Todavia, pesquisas anteriores apontam a necessidade de aprofundamento das técnicas e processos de desenvolvimento de jogos educativos, de modo que garantam a concepção de produtos adequados à demanda educacional.

A experiência aqui relatada apresenta-se como uma proposta inovadora e mais significativa na formação de alunos de qualquer segmento de ensino. Os resultados demonstram que mesmo sem ter o conhecimento específico sobre a criação de jogos 
digitais, os estudantes conseguem conceber o Game Design de seus próprios jogos, desde que recebem uma mediação adequada.

Como principal benefício proporcionado pelo CGD destaca-se a criação do Game Design de jogos digitais educativos, em curto prazo, de maneira criativa e colaborativa. O CGD proporciona uma experiência formativa do aluno, que motivado pelo desafio de produzir um jogo aprende não só os conceitos relacionados ao Game Design e ao processo de desenvolvimento, como também os conteúdos curriculares envolvidos.

Consideramos esse trabalho uma experiência piloto válida e que os resultados alcançados servem como subsidio para a realização de novos experimentos. Como futuros trabalhos, pretendemos melhorar o CGD, validando-o através de outras experiências.

\section{Referências}

ADAMS, Ernest; ROLLINGS, Andrew. Fundamental of Game Design. New Jersey: Pearson Prentice Hall. 2007.

ARRUDA, Eucidio Pimenta. Fundamentos para o desenvolvimento de jogos digitais. Porto Alegre: Bookman. 2014.

BRATHWAITE, Brenda; SCHREIBER, Iran. Challenges for Game Designers: nondigital exercicies for vídeo game designers. Boston: Cengage Leaning. 2009.

BROWN, T.; WYATT, J. Design thinking for Social Innovation. Stanford Social Innovation Review. California: Leland Stanford Jr. University. 2010.

CHANDLER, H. M. Manual de Produção de Jogos Digitais. Porto Alegre - RS: Bookman. 2012.

CROSS. Nigel. Design Thinking: Understanding how designers think and work. Nova Iorque. 2011.

EDUCADIGITAL, Instituto. Design Thinking para Educadores. Versão em Português 2013: Instituto Educadigital. Disponível em: http://www.dtparaeducadores.org.br/site/download-de-capitulos/. Acesso em: $10 / 03 / 2018$.

JEWELL, Desirre. Design Thinking in Education and Beyond. Higher Education, PreK-12 Education, Professional. 2016. Disponível em: http://www.pearsoned.com/education-blog/gamedesign-thinking-in-education-andbeyond/. Acesso em: 18/03/2018.

LOCKWOOD, T. Design thinking: Integrating innovation, customer experience, and brand value. New York: Allworth Press. 2009.

MARINHO, F. C. V., GIANNELLA, T. R. and STRUCHINER, M. Estudantes do Ensino Básico Como Desenvolvedores de Jogos Digitais: Contextos Autênticos de Aprendizagem para Educação em Ciências e Matemática, Em: Atas do VIII Encontro Nacional de Pesquisa em Educação em Ciências, Campinas, São Paulo. 2011. 
MARTINS, Daniel de S.; XAVIER Guilherme; DIAS, Cynthia. Game design thinking: propostas lúdicas de letramento digital infantil Simpósio Brasileiro de Games SBGAMES. Curitiba/PR. 2017.

MASTROCOLA, Vicente Martin. Game Design: modelo de negócio e processos criativos: um trajeto do protótipo ao jogo produzido. São Paulo: Cengage Learning. 2015.

MURAKAMI, L.C.; JUNIOR, Antônio José; SABINO, R. Felipe; MACEDO, D. Almeida. Design Thinking como metodologia alternativa para o desenvolvimento de jogos sérios. Nuevas Ideas en Informática Educativa. TISE 2014.

NOGUEIRA, A. S.; GALDINO, A. L. Games como agentes motivadores na educação. In: VIII Seminário Jogos eletrônicos, Educação e Comunicação. Salvador/BA. 2012.

PRENSKY, M. Digital natives, digital immigrants, part 1. On the horizon, v. 9, n.5, p. 1-6. 2001.

PRENSKY, M. Não me atrapalhe, mãe - eu estou aprendendo. São Paulo: Phorte. 2010.

SAVI, Rafael; ULBRICHT, Vania Jogos digitais educacionais: benefícios e desafios. Centro Disciplinar de Novas Tecnologias na Educação - CINTED - UFRGS. V6. N1. 2008. Disponível em: http://seer.ufrgs.br/index.php/renote/article/view/14405/8310. Acesso em: 12/04/2018.

SILVA, Alessandra M; CORREA, Ana C.S.; FILHO, José M.; SOUZA, Ricardo A. Aplicação do Design Thinking em um Problema Educacional: Um Relato de Experiência. V Congresso Brasileiro de Informática na Educação. XXII Workshop de Informática na Escola - WIE. Porto Alegre/RS. 2016.

SILVA, Isabel C. S.; BITTENCOURT, João Ricardo. Proposta de metodologia para o ensino e desenvolvimento de jogos digitais baseada em design thinking. Educação Gráfica, Volume 20, Número 03. 2017.

SILVA, Thiago Reis. Um Relato de Experiência da Aplicação de Gamificação e Game Design com Professores. VI Congresso Brasileiro de Informática na Educação. XXIII Workshop de Informática na Escola - WIE. São Paulo/SP. 2017.

SUTHERLAND, Jeff. Scrum - A Arte de Faze o Dobro de Trabalho na Metade do Tempo. $1^{a}$ ed. Leya Brasil, São Paulo. 2014.

VARGAS, Verônica C.L. Uma extensão do Design Thinking Canvas com foco em modelos de negócio para a indústria de games. Dissertação de Mestrado em Design e Ergonomia. Centro de Artes e Comunicação. UFPE. 2015. 